Acta Crystallographica Section E

Structure Reports

Online

ISSN 1600-5368

Editors: W. Clegg and D. G. Watson

\title{
2-(1,4-Dioxo-1,4-dihydro-2-naphthyl)-2-methylpropanoic acid
}

\section{Kieran J. Dempster-Rivett, Lyndsay Main, Brian K. Nicholson and William A.} Denny

Copyright $\odot$ International Union of Crystallography

Author(s) of this paper may load this reprint on their own web site or institutional repository provided that this cover page is retained. Republication of this article or its storage in electronic databases other than as specified above is not permitted without prior permission in writing from the IUCr.

For further information see http://journals.iucr.org/services/authorrights.html 


\section{organic compounds}

Acta Crystallographica Section E

\section{Structure Reports}

Online

ISSN 1600-5368

\section{2-(1,4-Dioxo-1,4-dihydro-2-naphthyl)- 2-methylpropanoic acid}

\section{Kieran J. Dempster-Rivett, ${ }^{a}$ Lyndsay Main, ${ }^{a}$ Brian K. Nicholson $^{a *}$ and William A. Denny ${ }^{b}$}

${ }^{a}$ Chemistry Department, University of Waikato, Private Bag 3105, Hamilton 3240, New Zealand, and ${ }^{\mathbf{b}}$ Auckland Cancer Society Research Centre, School of Medicine, University of Auckland, Private Bag 92019, Auckland, New Zealand

Correspondence e-mail: b.nicholson@waikato.ac.nz

Received 18 September 2007; accepted 1 October 2007

Key indicators: single-crystal X-ray study; $T=200 \mathrm{~K}$; mean $\sigma(\mathrm{C}-\mathrm{C})=0.002 \AA$; $R$ factor $=0.033 ; w R$ factor $=0.092 ;$ data-to-parameter ratio $=12.7$.

The sterically crowded title compound, $\mathrm{C}_{14} \mathrm{H}_{12} \mathrm{O}_{4}$, crystallizes as centrosymmetric hydrogen-bonded dimers involving the carboxyl groups. The naphthoquinone ring system is folded by $11.5(1)^{\circ}$ about a vector joining the 1,4-C atoms, and the quinone $\mathrm{O}$ atoms are displaced from the ring plane, presumably because of steric interactions with the bulky substituent.

\section{Related literature}

For synthesis details, see: Petersen \& Heitzer (1972). For related studies of $o$-hydroxycinnamic acids and benzoquinones, see: Karle \& Karle (1972); Wang et al. (1996). For related naphthoquinone structures, see: Gaultier \& Hauw (1965); Gaultier et al. (1971).<smiles>CC(C)(C(=O)O)C1=CC(=O)c2ccccc2C1=O</smiles>

\section{Experimental}

Crystal data

$$
\begin{aligned}
& \mathrm{C}_{14} \mathrm{H}_{12} \mathrm{O}_{4} \\
& M_{r}=244.24 \\
& \text { Monoclinic, } P 2_{1} / c \\
& a=8.3070(2) \AA \\
& b=6.5027(2) \AA \\
& c=22.0392(6) \AA \\
& \beta=97.219(1)^{\circ}
\end{aligned}
$$

\section{Data collection}

Siemens SMART CCD diffractometer

Absorption correction: multi-scan (SADABS; Sheldrick, 2003)

$T_{\min }=0.790, T_{\max }=0.990$

\section{Refinement}

$R\left[F^{2}>2 \sigma\left(F^{2}\right)\right]=0.033$

$w R\left(F^{2}\right)=0.092$

$S=1.03$

2145 reflections

169 parameters

\author{
$V=1181.07(6) \AA^{3}$ \\ $Z=4$ \\ Mo $K \alpha$ radiation \\ $\mu=0.10 \mathrm{~mm}^{-1}$ \\ $T=200(2) \mathrm{K}$ \\ $0.38 \times 0.18 \times 0.10 \mathrm{~mm}$
}

Table 1

Hydrogen-bond geometry $\left(\AA{ }^{\circ}\right)$.

\begin{tabular}{lllll}
\hline$D-\mathrm{H} \cdots A$ & $D-\mathrm{H}$ & $\mathrm{H} \cdots A$ & $D \cdots A$ & $D-\mathrm{H} \cdots A$ \\
\hline $\mathrm{O} 3-\mathrm{H} 1 \cdots \mathrm{O} 44^{\mathrm{i}}$ & $0.935(19)$ & $1.711(19)$ & $2.6455(15)$ & $176.5(16)$ \\
\hline
\end{tabular}

Symmetry code: (i) $-x,-y+1,-z$.

Data collection: SMART (Bruker, 2001); cell refinement: SAINT (Bruker, 2001); data reduction: SAINT; program(s) used to solve structure: SHELXS97 (Sheldrick, 1997); program(s) used to refine structure: SHELXL97 (Sheldrick, 1997); molecular graphics: ORTEP-3 for Windows (Farrugia, 1997); software used to prepare material for publication: WinGX (Farrugia, 1999).

We thank Dr Jan Wikaira, University of Canterbury, for collection of X-ray intensity data.

Supplementary data and figures for this paper are available from the IUCr electronic archives (Reference: BI2238).

\section{References}

Bruker (2001). SMART and SAINT. Bruker AXS Inc., Madison, Wisconsin, USA.

Farrugia, L. J. (1997). J. Appl. Cryst. 30, 565.

Farrugia, L. J. (1999). J. Appl. Cryst. 32, 837-838.

Gaultier, J., Hauw, C., Housty, J. \& Schvoerer, M. (1971). C. R. Acad. Sci. C, 273, 956-958.

Gaultier, J. \& Hauw, C. (1965). Acta Cryst. 18, 179-183.

Karle, J. M. \& Karle, I. M. (1972). J. Am. Chem. Soc. 94, 9182-9189.

Petersen, S. \& Heitzer, H. (1972). Liebigs Ann. Chem. 764, 36-43.

Sheldrick, G. M. (1997). SHELXS97 and SHELXL97. University of Göttingen, Germany.

Sheldrick, G. M. (2003). SADABS. University of Göttingen, Germany.

Wang, B., Nicolaou, M. G., Liu, S. \& Borchardt, R. T. (1996). Bioorg. Chem. 24, $39-49$. 


\section{supplementary materials}




\section{supplementary materials}

Acta Cryst. (2007). E63, o4251 [ doi:10.1107/S1600536807048131 ]

\section{2-(1,4-Dioxo-1,4-dihydro-2-naphthyl)-2-methylpropanoic acid}

\section{K. J. Dempster-Rivett, L. Main, B. K. Nicholson and W. A. Denny}

\section{Comment}

The title compound was prepared as part of a study to examine constrained conformational effects on lactonization of naphthohydroquinone carboxylic acid derivatives for comparison with related work on sterically crowded $o$-hydroxycinnamic acids and benzoquinone analogues with a "trimethyl-lock" effect (Karle \& Karle, 1972; Wang et al., 1996).

The compound crystallizes as a dimer formed about an inversion centre by $\mathrm{O}-\mathrm{H} \cdots \mathrm{O}$ hydrogen bonding between the carboxyl groups. The naphthoquinone ring is folded by $11.5(1)^{\circ}$ about a vector joining the 1 ,4-carbon atoms and the quinone $\mathrm{O}$ atoms are displaced from the ring plane, presumably because of steric interactions with the adjacent geminal dimethyl groups, and with the carboxyl group. Even with the bending, there is still a close intramolecular contact between $\mathrm{O} 1$ and $\mathrm{O} 3$

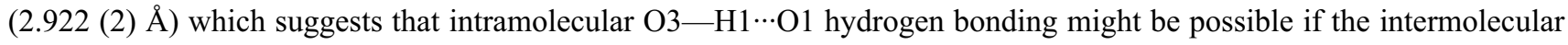
dimer form was not preferred. Naphthoquinone itself, and other 2-substituted naphthoquinones (e.g. the 2-iodo derivative) are planar (Gaultier and Hauw, 1965; Gaultier et al., 1971). However, benzoquinones with tri-substituted carbon atoms in the 2-position also show displacement of the quinone $\mathrm{O}$ atoms from the plane of the ring (Wang et al., 1996).

\section{Experimental}

The title compound was prepared using the method of Petersen \& Heitzer (1972). Crystals suitable for X-ray analysis were grown from a solution in toluene.

\section{Refinement}

The carboxyl $\mathrm{H}$ atom was located in a penultimate difference Fourier map and its position was refined freely with $U_{\text {iso }}=$ $0.05 \AA^{2}$. All other $\mathrm{H}$ atoms were positioned geometrically and refined using a riding model with $\mathrm{C}-\mathrm{H}=0.95 \AA, U_{\text {iso }}(\mathrm{H})=$ $1.2 U_{\text {eq }}(\mathrm{C})$ for aromatic $\mathrm{H}$, and with $\mathrm{C}-\mathrm{H}=0.96 \AA, U_{\text {iso }}(\mathrm{H})=1.5 U_{\text {eq }}(\mathrm{C})$ for the methyl groups.

\section{Figures}

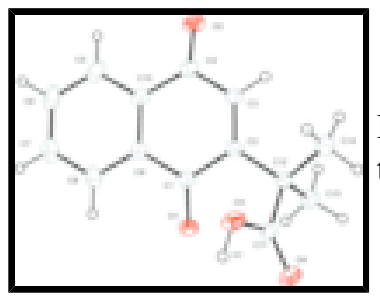

Fig. 1. The molecular structure of the title compound with displacement parameters drawn at the $30 \%$ probability level for non-H atoms.

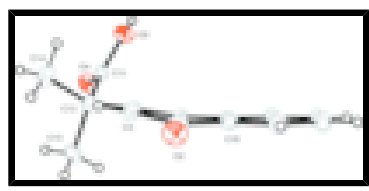

Fig. 2. Side-on view showing the folding of the aromatic ring. 


\section{supplementary materials}

\section{2-(1,4-Dioxo-1,4-dihydro-2-naphthyl)-2-methylpropanoic acid}

$$
\begin{aligned}
& \text { Crystal data } \\
& \mathrm{C}_{14} \mathrm{H}_{12} \mathrm{O}_{4} \\
& M_{r}=244.24 \\
& \text { Monoclinic, } P 2{ }_{1} / c \\
& \text { Hall symbol: }-\mathrm{P} 2 \mathrm{ybc} \\
& a=8.3070(2) \AA \\
& b=6.5027(2) \AA \\
& c=22.0392(6) \AA \\
& \beta=97.219(1)^{\circ} \\
& V=1181.07(6) \AA^{3} \\
& Z=4
\end{aligned}
$$

\section{Data collection}

Siemens SMART CCD

diffractometer

Radiation source: fine-focus sealed tube

Monochromator: graphite

$T=200(2) \mathrm{K}$

$\omega$ scans

Absorption correction: multi-scan

(SADABS; Sheldrick, 2003)

$T_{\min }=0.790, T_{\max }=0.990$

6372 measured reflections

\section{Refinement}

Refinement on $F^{2}$

Least-squares matrix: full

$R\left[F^{2}>2 \sigma\left(F^{2}\right)\right]=0.033$

$w R\left(F^{2}\right)=0.092$

$S=1.03$

2145 reflections

169 parameters

Primary atom site location: structure-invariant direct methods

Secondary atom site location: difference Fourier map
$F_{000}=512$

$D_{\mathrm{x}}=1.374 \mathrm{Mg} \mathrm{m}^{-3}$

Mo $K \alpha$ radiation

$\lambda=0.71073 \AA$

Cell parameters from 3955 reflections

$\theta=2-25^{\circ}$

$\mu=0.10 \mathrm{~mm}^{-1}$

$T=200$ (2) K

Needle, yellow

$0.38 \times 0.18 \times 0.10 \mathrm{~mm}$

2145 independent reflections

1722 reflections with $I>2 \sigma(I)$

$R_{\text {int }}=0.021$

$\theta_{\max }=25.4^{\circ}$

$\theta_{\min }=1.9^{\circ}$

$h=-9 \rightarrow 9$

$k=0 \rightarrow 7$

$l=0 \rightarrow 26$

Hydrogen site location: inferred from neighbouring sites

$\mathrm{H}$ atoms treated by a mixture of independent and constrained refinement

$w=1 /\left[\sigma^{2}\left(F_{\mathrm{o}}{ }^{2}\right)+(0.0458 P)^{2}+0.2618 P\right]$

where $P=\left(F_{\mathrm{o}}{ }^{2}+2 F_{\mathrm{c}}{ }^{2}\right) / 3$

$(\Delta / \sigma)_{\max }<0.001$

$\Delta \rho_{\max }=0.22$ e $\AA^{-3}$

$\Delta \rho_{\min }=-0.17$ e $\AA^{-3}$

Extinction correction: SHELXL97 (Sheldrick, 1997),

$\mathrm{Fc}^{*}=\mathrm{kFc}\left[1+0.001 \mathrm{xFc}^{2} \lambda^{3} / \sin (2 \theta)\right]^{-1 / 4}$

Extinction coefficient: 0.014 (2) 


\section{supplementary materials}

Fractional atomic coordinates and isotropic or equivalent isotropic displacement parameters $\left(\AA^{2}\right)$

\begin{tabular}{|c|c|c|c|c|}
\hline & $x$ & $y$ & $z$ & $U_{\text {iso }} * / U_{\text {eq }}$ \\
\hline $\mathrm{O} 1$ & $0.22816(14)$ & $0.56642(15)$ & $0.14194(5)$ & $0.0427(3)$ \\
\hline $\mathrm{O} 2$ & $0.40002(14)$ & -0.08507 (17) & $0.27913(5)$ & $0.0483(3)$ \\
\hline $\mathrm{O} 3$ & $0.03089(13)$ & $0.29681(18)$ & $0.05640(5)$ & $0.0412(3)$ \\
\hline $\mathrm{O} 4$ & $0.19621(13)$ & $0.46089(17)$ & $0.00002(5)$ & 0.0435 \\
\hline $\mathrm{H} 1$ & $-0.046(2)$ & $0.386(3)$ & $0.0360(8)$ & $0.050^{*}$ \\
\hline $\mathrm{C} 1$ & $0.24736(16)$ & $0.4101(2)$ & $0.17254(6)$ & $0.0307(3)$ \\
\hline $\mathrm{C} 2$ & $0.29793(16)$ & $0.2139(2)$ & $0.14460(6)$ & $0.0289(3)$ \\
\hline $\mathrm{C} 3$ & $0.33983(17)$ & $0.0523(2)$ & $0.18060(6)$ & $0.0330(3)$ \\
\hline H3 & 0.3707 & -0.0713 & 0.1622 & $0.040^{*}$ \\
\hline $\mathrm{C} 4$ & $0.34047(17)$ & $0.0569(2)$ & $0.24761(6)$ & $0.0350(3)$ \\
\hline $\mathrm{C} 5$ & $0.23413(19)$ & $0.2327(3)$ & $0.33547(7)$ & 0.0469 (4) \\
\hline H5 & 0.2649 & 0.1169 & 0.3605 & $0.056^{*}$ \\
\hline C6 & $0.1598(2)$ & $0.3998(3)$ & $0.35918(7)$ & $0.0540(5)$ \\
\hline H6 & 0.1387 & 0.3978 & 0.4006 & $0.065^{*}$ \\
\hline $\mathrm{C} 7$ & $0.11625(19)$ & $0.5692(3)$ & $0.32321(8)$ & $0.0507(5)$ \\
\hline $\mathrm{H} 7$ & 0.0662 & 0.6835 & 0.3401 & $0.061^{*}$ \\
\hline $\mathrm{C} 8$ & $0.14484(18)$ & $0.5740(3)$ & $0.26257(7)$ & $0.0400(4)$ \\
\hline $\mathrm{H} 8$ & 0.1146 & 0.6912 & 0.2380 & $0.048^{*}$ \\
\hline C9 & $0.21794(16)$ & $0.4066(2)$ & $0.23789(6)$ & $0.0328(3)$ \\
\hline $\mathrm{C} 10$ & $0.26363(16)$ & $0.2354(2)$ & $0.27450(6)$ & $0.0351(3)$ \\
\hline C11 & $0.17484(18)$ & $0.3414(2)$ & $0.04156(6)$ & $0.0322(3)$ \\
\hline $\mathrm{C} 12$ & $0.31285(17)$ & $0.2190(2)$ & $0.07637(6)$ & $0.0327(3)$ \\
\hline $\mathrm{C} 13$ & $0.47669(18)$ & $0.3179(3)$ & $0.06739(7)$ & $0.0423(4)$ \\
\hline $\mathrm{H} 13 \mathrm{~A}$ & 0.4896 & 0.3180 & 0.0238 & $0.063^{*}$ \\
\hline H13B & 0.5651 & 0.2390 & 0.0901 & $0.063^{*}$ \\
\hline H13C & 0.4797 & 0.4597 & 0.0826 & $0.063^{*}$ \\
\hline $\mathrm{C} 14$ & $0.3015(2)$ & $0.0004(3)$ & $0.04859(7)$ & 0.0491 \\
\hline $\mathrm{H} 14 \mathrm{~A}$ & 0.3959 & -0.0803 & 0.0658 & $0.074 *$ \\
\hline H14B & 0.2993 & 0.0095 & 0.0041 & $0.074 *$ \\
\hline $\mathrm{H} 14 \mathrm{C}$ & 0.2021 & -0.0665 & 0.0582 & $0.074 *$ \\
\hline
\end{tabular}

Atomic displacement parameters $\left(\hat{A}^{2}\right)$

$\begin{array}{lllllll} & U^{11} & U^{22} & U^{33} & U^{12} & U^{13} & U^{23} \\ \text { O1 } & 0.0608(7) & 0.0313(6) & 0.0360(6) & 0.0057(5) & 0.0062(5) & 0.0046(4) \\ \text { O2 } & 0.0507(7) & 0.0516(7) & 0.0408(6) & 0.0089(5) & -0.0010(5) & 0.0170(5) \\ \text { O3 } & 0.0335(6) & 0.0501(7) & 0.0389(6) & -0.0016(5) & 0.0012(4) & 0.0127(5) \\ \text { O4 } & 0.0414(6) & 0.0559(7) & 0.0333(6) & 0.0028(5) & 0.0054(4) & 0.0165(5) \\ \text { C1 } & 0.0292(7) & 0.0327(8) & 0.0294(7) & -0.0012(6) & 0.0004(5) & -0.0002(6) \\ \text { C2 } & 0.0273(7) & 0.0312(7) & 0.0277(7) & -0.0016(5) & 0.0013(5) & -0.0001(6) \\ \text { C3 } & 0.0347(7) & 0.0313(7) & 0.0322(8) & 0.0009(6) & 0.0009(6) & 0.0019(6) \\ \text { C4 } & 0.0300(7) & 0.0400(8) & 0.0334(8) & -0.0016(6) & -0.0016(6) & 0.0085(6) \\ \text { C5 } & 0.0378(9) & 0.0710(12) & 0.0310(8) & 0.0018(8) & 0.0008(6) & 0.0071(8) \\ \text { C6 } & 0.0410(9) & 0.0934(14) & 0.0277(8) & 0.0036(9) & 0.0052(7) & -0.0085(9)\end{array}$




\section{supplementary materials}

$\begin{array}{lllllll}\text { C7 } & 0.0377(9) & 0.0736(12) & 0.0407(9) & 0.0059(8) & 0.0044(7) & -0.0174(9) \\ \text { C8 } & 0.0339(8) & 0.0476(9) & 0.0382(8) & 0.0022(7) & 0.0030(6) & -0.0070(7) \\ \text { C9 } & 0.0273(7) & 0.0399(8) & 0.0305(7) & -0.0021(6) & 0.0015(5) & -0.0024(6) \\ \text { C10 } & 0.0277(7) & 0.0485(9) & 0.0280(7) & -0.0017(6) & -0.0003(6) & 0.0018(6) \\ \text { C11 } & 0.0369(8) & 0.0352(8) & 0.0242(7) & -0.0020(6) & 0.0025(5) & -0.0009(6) \\ \text { C12 } & 0.0381(8) & 0.0339(8) & 0.0261(7) & 0.0033(6) & 0.0034(6) & 0.0006(6) \\ \text { C13 } & 0.0370(8) & 0.0554(10) & 0.0351(8) & 0.0052(7) & 0.0071(6) & 0.0072(7) \\ \text { C14 } & 0.0724(12) & 0.0417(9) & 0.0321(8) & 0.0088(8) & 0.0024(7) & -0.0062(7)\end{array}$

Geometric parameters $\left(A,{ }^{\circ}\right)$

\begin{tabular}{|c|c|c|c|}
\hline $\mathrm{O} 1-\mathrm{C} 1$ & $1.2193(17)$ & $\mathrm{C} 6-\mathrm{H} 6$ & 0.950 \\
\hline $\mathrm{O} 2-\mathrm{C} 4$ & $1.2219(17)$ & $\mathrm{C} 7-\mathrm{C} 8$ & $1.387(2)$ \\
\hline $\mathrm{O} 3-\mathrm{C} 11$ & $1.3113(17)$ & $\mathrm{C} 7-\mathrm{H} 7$ & 0.950 \\
\hline $\mathrm{O} 3-\mathrm{H} 1$ & $0.935(19)$ & $\mathrm{C} 8-\mathrm{C} 9$ & $1.391(2)$ \\
\hline $\mathrm{O} 4-\mathrm{C} 11$ & $1.2303(17)$ & $\mathrm{C} 8-\mathrm{H} 8$ & 0.950 \\
\hline $\mathrm{C} 1-\mathrm{C} 9$ & $1.4910(19)$ & $\mathrm{C} 9-\mathrm{C} 10$ & $1.399(2)$ \\
\hline $\mathrm{C} 1-\mathrm{C} 2$ & $1.4991(19)$ & $\mathrm{C} 11-\mathrm{C} 12$ & $1.522(2)$ \\
\hline $\mathrm{C} 2-\mathrm{C} 3$ & $1.3364(19)$ & $\mathrm{C} 12-\mathrm{C} 13$ & $1.540(2)$ \\
\hline $\mathrm{C} 2-\mathrm{C} 12$ & $1.5246(18)$ & $\mathrm{C} 12-\mathrm{C} 14$ & $1.546(2)$ \\
\hline $\mathrm{C} 3-\mathrm{C} 4$ & $1.476(2)$ & $\mathrm{C} 13-\mathrm{H} 13 \mathrm{~A}$ & 0.980 \\
\hline $\mathrm{C} 3-\mathrm{H} 3$ & 0.950 & $\mathrm{C} 13-\mathrm{H} 13 \mathrm{~B}$ & 0.980 \\
\hline $\mathrm{C} 4-\mathrm{C} 10$ & $1.484(2)$ & $\mathrm{C} 13-\mathrm{H} 13 \mathrm{C}$ & 0.980 \\
\hline $\mathrm{C} 5-\mathrm{C} 6$ & $1.384(3)$ & $\mathrm{C} 14-\mathrm{H} 14 \mathrm{~A}$ & 0.980 \\
\hline $\mathrm{C} 5-\mathrm{C} 10$ & $1.396(2)$ & C14-H14B & 0.980 \\
\hline $\mathrm{C} 5-\mathrm{H} 5$ & 0.950 & $\mathrm{C} 14-\mathrm{H} 14 \mathrm{C}$ & 0.980 \\
\hline $\mathrm{C} 6-\mathrm{C} 7$ & $1.378(3)$ & & \\
\hline $\mathrm{C} 11-\mathrm{O} 3-\mathrm{H} 1$ & $109.5(10)$ & $\mathrm{C} 8-\mathrm{C} 9-\mathrm{C} 1$ & $119.91(13)$ \\
\hline $\mathrm{O} 1-\mathrm{C} 1-\mathrm{C} 9$ & $121.39(13)$ & $\mathrm{C} 10-\mathrm{C} 9-\mathrm{C} 1$ & $120.24(13)$ \\
\hline $\mathrm{O} 1-\mathrm{C} 1-\mathrm{C} 2$ & $120.41(12)$ & $\mathrm{C} 5-\mathrm{C} 10-\mathrm{C} 9$ & $119.82(14)$ \\
\hline $\mathrm{C} 9-\mathrm{C} 1-\mathrm{C} 2$ & $118.18(12)$ & $\mathrm{C} 5-\mathrm{C} 10-\mathrm{C} 4$ & $120.75(14)$ \\
\hline $\mathrm{C} 3-\mathrm{C} 2-\mathrm{C} 1$ & $119.38(12)$ & $\mathrm{C} 9-\mathrm{C} 10-\mathrm{C} 4$ & $119.42(12)$ \\
\hline $\mathrm{C} 3-\mathrm{C} 2-\mathrm{C} 12$ & $123.74(12)$ & $\mathrm{O} 4-\mathrm{C} 11-\mathrm{O} 3$ & $123.14(13)$ \\
\hline $\mathrm{C} 1-\mathrm{C} 2-\mathrm{C} 12$ & $116.64(11)$ & $\mathrm{O} 4-\mathrm{C} 11-\mathrm{C} 12$ & $122.37(13)$ \\
\hline $\mathrm{C} 2-\mathrm{C} 3-\mathrm{C} 4$ & $123.10(13)$ & $\mathrm{O} 3-\mathrm{C} 11-\mathrm{C} 12$ & $114.29(12)$ \\
\hline $\mathrm{C} 2-\mathrm{C} 3-\mathrm{H} 3$ & 118.4 & $\mathrm{C} 11-\mathrm{C} 12-\mathrm{C} 2$ & $111.05(11)$ \\
\hline $\mathrm{C} 4-\mathrm{C} 3-\mathrm{H} 3$ & 118.4 & $\mathrm{C} 11-\mathrm{C} 12-\mathrm{C} 13$ & $109.80(12)$ \\
\hline $\mathrm{O} 2-\mathrm{C} 4-\mathrm{C} 3$ & $120.25(14)$ & $\mathrm{C} 2-\mathrm{C} 12-\mathrm{C} 13$ & $108.57(11)$ \\
\hline $\mathrm{O} 2-\mathrm{C} 4-\mathrm{C} 10$ & 121.85 & $\mathrm{C} 11-\mathrm{C} 12-\mathrm{C} 14$ & $106.06(12)$ \\
\hline $\mathrm{C} 3-\mathrm{C} 4-\mathrm{C} 10$ & $117.88(12)$ & $\mathrm{C} 2-\mathrm{C} 12-\mathrm{C} 14$ & $111.23(12)$ \\
\hline $\mathrm{C} 6-\mathrm{C} 5-\mathrm{C} 10$ & $119.63(16)$ & $\mathrm{C} 13-\mathrm{C} 12-\mathrm{C} 14$ & $110.11(13)$ \\
\hline $\mathrm{C} 6-\mathrm{C} 5-\mathrm{H} 5$ & 120.2 & $\mathrm{C} 12-\mathrm{C} 13-\mathrm{H} 13 \mathrm{~A}$ & 109.5 \\
\hline $\mathrm{C} 10-\mathrm{C} 5-\mathrm{H} 5$ & 120.2 & $\mathrm{C} 12-\mathrm{C} 13-\mathrm{H} 13 \mathrm{~B}$ & 109.5 \\
\hline $\mathrm{C} 7-\mathrm{C} 6-\mathrm{C} 5$ & $120.48(14)$ & $\mathrm{H} 13 \mathrm{~A}-\mathrm{C} 13-\mathrm{H} 13 \mathrm{~B}$ & 109.5 \\
\hline $\mathrm{C} 7-\mathrm{C} 6-\mathrm{H} 6$ & 119.8 & $\mathrm{C} 12-\mathrm{C} 13-\mathrm{H} 13 \mathrm{C}$ & 109.5 \\
\hline $\mathrm{C} 5-\mathrm{C} 6-\mathrm{H} 6$ & 119.8 & $\mathrm{H} 13 \mathrm{~A}-\mathrm{C} 13-\mathrm{H} 13 \mathrm{C}$ & 109.5 \\
\hline $\mathrm{C} 6-\mathrm{C} 7-\mathrm{C} 8$ & $120.53(16)$ & $\mathrm{H} 13 \mathrm{~B}-\mathrm{C} 13-\mathrm{H} 13 \mathrm{C}$ & 109.5 \\
\hline $\mathrm{C} 6-\mathrm{C} 7-\mathrm{H} 7$ & 119.7 & $\mathrm{C} 12-\mathrm{C} 14-\mathrm{H} 14 \mathrm{~A}$ & 109.5 \\
\hline $\mathrm{C} 8-\mathrm{C} 7-\mathrm{H} 7$ & 119.7 & $\mathrm{C} 12-\mathrm{C} 14-\mathrm{H} 14 \mathrm{~B}$ & 109.5 \\
\hline
\end{tabular}




\section{supplementary materials}

$\begin{array}{ll}\mathrm{C} 7-\mathrm{C} 8-\mathrm{C} 9 & 119.69(16) \\ \mathrm{C} 7-\mathrm{C} 8-\mathrm{H} 8 & 120.2 \\ \mathrm{C} 9-\mathrm{C} 8-\mathrm{H} 8 & 120.2 \\ \mathrm{C} 8-\mathrm{C} 9-\mathrm{C} 10 & 119.84(13)\end{array}$

$\mathrm{H} 14 \mathrm{~A}-\mathrm{C} 14-\mathrm{H} 14 \mathrm{~B}$

109.5

$\mathrm{C} 12-\mathrm{C} 14-\mathrm{H} 14 \mathrm{C}$

109.5

$\mathrm{H} 14 \mathrm{~A}-\mathrm{C} 14-\mathrm{H} 14 \mathrm{C}$

109.5

$\mathrm{C} 8-\mathrm{C} 9-\mathrm{C} 10$

$\mathrm{H} 14 \mathrm{~B}-\mathrm{C} 14-\mathrm{H} 14 \mathrm{C}$

109.5

Hydrogen-bond geometry $\left(\AA,{ }^{\circ}\right)$

$D-\mathrm{H} \cdots A$

$D-\mathrm{H}$

$\mathrm{H} \cdots A$

$D \cdots A$

$D-\mathrm{H} \cdots A$

$\mathrm{O} 3-\mathrm{H} 1 \cdots \mathrm{O} 4^{\mathrm{i}}$

0.935 (19)

$1.711(19)$

2.6455 (15)

176.5 (16)

Symmetry codes: (i) $-x,-y+1,-z$. 
Fig. 1

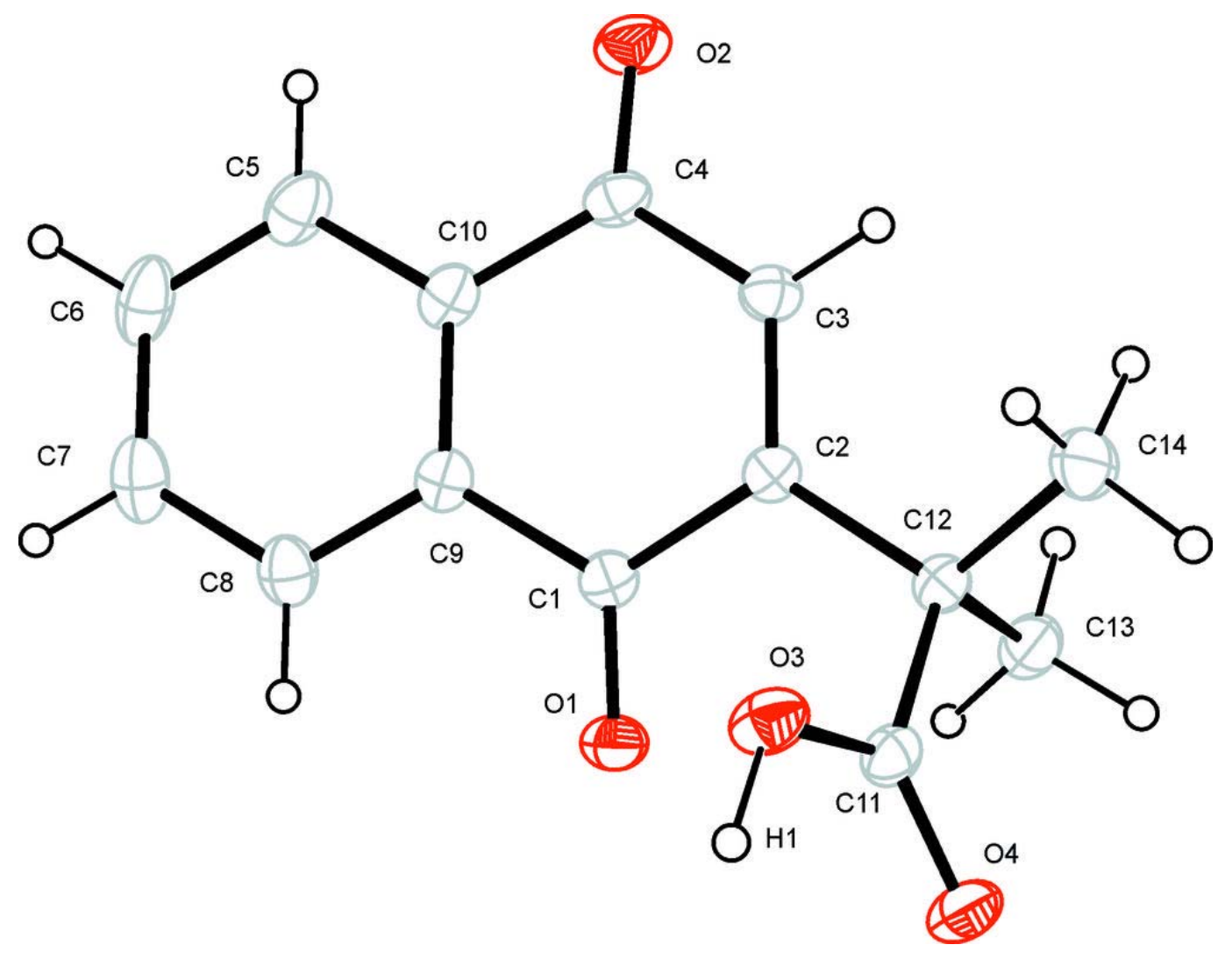


Fig. 2

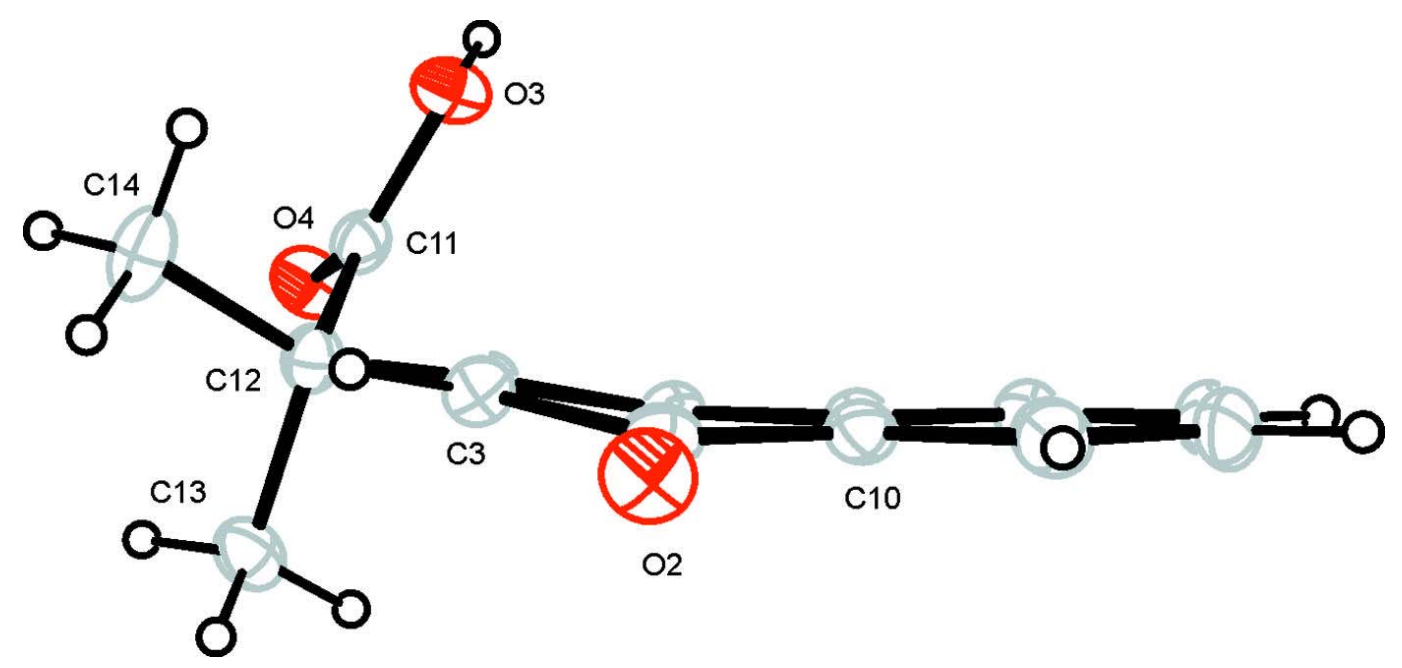

